\title{
Costi diretti e indiretti assorbiti dalle patologie HCV-indotte in Italia: stima basata su una metodologia probabilistica di Cost of Illness Cost of illness probabilistic methodolo...
}

\author{
ARTICLE in PHARMACOECONOMICS - ITALIAN RESEARCH ARTICLES · JANUARY 2014
}

Impact Factor: 3.34 · DOI: 10.1007/s40276-014-0023-9

CITATION

1

4 AUTHORS:

Francesco Saverio Mennini

University of Rome Tor Vergata

85 PUBLICATIONS 241 CITATIONS

SEE PROFILE

Alessandro Capone

Kingston University London

41 PUBLICATIONS 229 CITATIONS

SEE PROFILE
READS

45
Andrea Marcellusi

University of Rome Tor Vergata

64 PUBLICATIONS 172 CITATIONS

SEE PROFILE

Raffaella Viti

University of Rome Tor Vergata

9 PUBLICATIONS 5 CITATIONS

SEE PROFILE 


\title{
Costi diretti e indiretti assorbiti dalle patologie HCV-indotte in Italia: stima basata su una metodologia probabilistica di Cost of Illness
}

\author{
Cost of illness probabilistic methodology to assess direct and indirect costs \\ of HCV-related diseases in Italy
}

\author{
A. Marcellusi • R. Viti • A. Capone · F.S. Mennini
}

(C) Springer International Publishing Switzerland 2014

\begin{abstract}
Background The hepatitis C virus (HCV) induces several pathological conditions worldwide with a substantial medical and economic burden. The objective of this study was to estimate the average annual cost incurred by the National Health Service (NHS) as well as society due to HCV in Italy.

Methods A probabilistic incidence-based cost of illness model was developed to estimate an aggregate measure of the economic burden associated with $\mathrm{HCV}$-induced diseases either in terms of direct or indirect costs. Indirect costs were calculated on the basis of productivity lost according to the human capital approach. A systematic literature review was carried out to identify epidemiological and economic data which were subsequently used to inform the model. Furthermore, a one-way probabilistic sensitivity analysis with 5,000 Monte Carlo simulations was performed, in order to test the robustness of results and define the proper $95 \% \mathrm{CI}$.
\end{abstract}

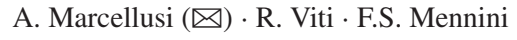

Economic Evaluation and HTA (CEIS-EEHTA)_IGF

Department, Faculty of Economics, University of Rome

"Tor Vergata", Via Columbia 2, 00133 Roma, Italia

e-mail: andrea.marcellusi@uniroma2.it

R. Viti

e-mail: raffaella.v@live.it
}

\section{A. Marcellusi}

Department of Demography, University of Rome "La Sapienza",

Roma, Italia

\section{A. Capone · F.S. Mennini}

Institute for Leadership and Management in Health, Kingston

University London, London, UK

\footnotetext{
A. Capone

e-mail: A.Capone@kingston.ac.uk

F.S. Mennini

e-mail: f.mennini@uniroma2.it
}

Results Overall, the total economic burden associated with HCV-induced diseases was estimated in $€ 1.05$ (95\% CI: $€ 0.61-€ 1.61)$ billion. A percentage equal to $61.4 \%$ was associated with indirect costs (95\% CI:€0.37-€0.99 billion) and $38,6 \%$ with direct costs (95\% CI: $€ 0.23-€ 0.63$ billion). For chronic hepatitis $\mathrm{C}$, cirrhosis, hepatocellular carcinoma (HCC), liver transplantation and death from causes related to $\mathrm{HCV}$ was estimated an average annual economic burden amounting to $€ 0.26$ (95\% CI: $€ 0.14-€ 0.41), € 0.55$ (95\% CI: €0.30-€0.87), €0.051 (95\% CI: €0.0001$€ 0.25) € 0.05$ (95\% CI: $€ 0.03-€ 0.08)$ and $€ 0.15$ (95\% CI: $€ 0.06-€ 0.27)$ billion, respectively.

Conclusions Italy is one of the European countries with the highest number of people with chronic HCV infection, the leading cause of cirrhosis, HCC and liver-related death. $\mathrm{HCV}$-induced diseases cause a significant cost for Italian NHS, especially for each case of liver transplantation. These highly debilitating and life-threatening complications generate a rather large amount of indirect costs for the Italian society as well.

\footnotetext{
Key-message

- Ad oggi, non esiste uno studio di prevalenza dell'infezione da HCV su un campione rappresentativo dell'intera popolazione Italiana né tantomeno è possibile stabilirne il peso economico per il Sistema sanitario e per la società.

- L'obiettivo di questa analisi era quello di sistematizzare le informazioni epidemiologiche ed economiche disponibili per il nostro paese e stimare il peso economico annuo sostenuto dalla società italiana (costi diretti sanitari e costi indiretti) per il monitoraggio, il trattamento e la gestione dei pazienti con infezione cronica da HCV.
} 
Il modello epidemiologico ha consentito di stabilire che il numero di pazienti prevalenti con diagnosi di patologie $\mathrm{HCV}$-indotte nel nostro paese dovrebbe corrispondere a 299.195 individui. Inoltre, lo studio ha permesso di calcolare l'onere economico assorbito dalle patologie HCV-indotte nel 2013, stimando un costo medio annuo di poco superiore ad 1,0 miliardo di Euro.

- Lo studio può essere ritenuto un'efficiente strumento di riferimento per i decisori pubblici che vogliano comprendere adeguatamente gli aspetti economici generati dalla gestione e dal trattamento delle patologie $\mathrm{HCV}$-indotte in Italia.

\section{Introduzione}

Il virus dell'epatite C (HCV) può essere trasmesso mediante esposizione a sangue o emoderivati infetti, più frequentemente in seguito a trasfusione di sangue o di fattori di coagulazione oppure al contatto con aghi ipodermici contaminati [1].

L'infezione da HCV è una delle emergenze sanitarie più pressanti, con una prevalenza globale di circa 170 milioni di persone cronicamente infette in tutto il mondo [2]. In Italia, la prevalenza di HCV (rilevata con test HCV-RNA) è generalmente superiore al 3\% nei soggetti nati prima del 1950 e aumenta progressivamente con l'età, mentre è considerevolmente più bassa nelle generazioni più giovani [3]. Attualmente, non è disponibile uno studio di prevalenza dell'infezione da HCV rappresentativo dell'intera popolazione Italiana [4]; tuttavia, sulla base di informazioni non omogeneamente distribuite e, pertanto, relativamente precise, lo European Centre for Disease Prevention and Control (ECDC)ha prodotto delle stime di prevalenza [5]. La prevalenza è, inoltre, più alta nelle aree meridionali e insulari rispetto a quelle del centro e del nord, variando rispettivamente dall' $8 \%$ al $2 \%$ [3].

Il problema dell'infezione da HCV risiede nella possibile evoluzione che si manifesta se nel soggetto infetto la malattia si protrae nel lungo periodo. Infatti, la storia naturale dell'infezione/malattia da virus dell'epatite $\mathrm{C}$ si compie nell'arco di decenni. Tra i pazienti che acquisiscono l'infezione da HCV, infatti, oltre il $90 \%$ diventa portatore cronico, sviluppando nel 65-95\% dei casi un'epatopatia cronica che rischia di evolvere nel 10-30\% dei casi in cirrosi nell'arco di 25-30 anni [6]. I soggetti cirrotici a loro volta hanno una probabilità dell'1-3\% per anno di sviluppare un epatocarcinoma (HCC) [6]. Questo, costringerà il Servizio Sanitario Nazionale (SSN) Italiano ad affrontare nei prossimi 5-10 anni un considerevole onere clinico determinato dalla gestione di questi pazienti $[3,7]$.

Alla notevole rilevanza epidemiologica corrisponde un sostanziale impatto economico delle malattie epatiche sul
SSN [4]. Alcuni studi hanno stimato un costo per il SSN pari a circa 500 milioni di euro [8]. Tuttavia, il potenziale impatto economico dell'infezione da HCV è molteplice: da una parte ci sono i costi diretti sanitari che comprendono le spese sostenute dal SSN per la gestione del paziente, dall'altra una persona affetta da infezione cronica, o che ha sviluppato una complicanza correlata, può essere incapace di lavorare a pieno regime, se non del tutto, con conseguente riduzione della produttività (costi indiretti). Ad oggi, questo aspetto è spesso trascurato e in Italia non sono stati stimati gli impatti economici di questa voce di costo.

L'obiettivo di questa analisi è quello di sistematizzare le informazioni epidemiologiche ed economiche disponibili per il nostro paese e stimare il peso economico annuo sostenuto dalla società italiana (costi diretti sanitari e costi indiretti) per il monitoraggio, il trattamento e la gestione dei pazienti con infezione cronica da $\mathrm{HCV}$.

\section{Metodi}

\subsection{Disegno dello studio}

Per stimare i costi annuali causati dall'infezione cronica da $\mathrm{HCV}$ in Italia è stato sviluppato un modello probabilistico di Cost of Illness (COI) capace di stimare la numerosità e la distribuzione dei pazienti per ciascuno stato di malattia HCV-indotto. Coerentemente con l'obiettivo dell' analisi, si è proceduto con la stima dei costi diretti sanitari e dei costi indiretti, intesi come perdita di produttività attribuibile alla malattia. Il modello è stato informato con dati derivati da una revisione sistematica della letteratura disponibile.

Per la stima dei costi diretti è stato utilizzato un approccio Bottom-Up, ampliamente utilizzato in analisi di questo genere [9, 10]. Questo sistema prevede la misura dei costi diretti della gestione del paziente, ottenuta moltiplicando il costo medio per la prevalenza della malattia stessa. Il costo medio diretto sanitario è stato stimato per HCV cronica, cirrosi, epatocarcinoma e trapianto di fegato, in funzione del diverso disease management utilizzato nelle differenti fasi evolutive della malattia.

Per la stima dei costi indiretti è stato impiegato lo $\mathrm{Hu}-$ man Capital Approach o Metodo del Capitale Umano; ciò equivale a misurare $\mathrm{i}$ costi in termini di deficit di guadagni dovuti alla perdita di produzione causata dalla malattia [11].

\subsection{Revisione sistematica della letteratura}

Per sviluppare il modello e reperire i parametri necessari per la ricostruzione della storia naturale della patologia, è stata effettuata una revisione sistematica della letteratura realizzata esaminando le pubblicazioni che analizzano, descrivono e confrontano studi epidemiologici e/o di valutazione economica concernenti l'infezione da HCV in Italia. 
La ricerca sistematica è stata effettuata considerando i seguenti motori di ricerca scientifica: MEDLINE (PubMed), l'Istituto nazionale di statistica (ISTAT), il Ministero della Salute, le Associazioni Nazionali e Internazionali di medici e pazienti (AISF, ${ }^{1}$ ECDC, SIT $^{2}$ EpaC Onlus) ed il sito EpiCentro, [il portale epidemiologico per la sanità pubblica, curato dall'Istituto superiore sanità (ISS) e dedicato alla sorveglianza e alla promozione della salute]. Inoltre, per identificare ulteriori pubblicazioni scientifiche italiane nel campo delle valutazioni economiche in ambito sanitario e garantire l'inclusione di tutti gli studi rilevanti per l'analisi sono state consultate le riviste italiane di economia sanitaria [PharmacoEconomics Italian Research Article (Springer), Farmeconomia e Percorsi Terapeutici (JournalSeek) e Il sole 24 ore-Sanità]. Giornali peer-reviewed, convegni, congressi e altre fonti disponibili in internet sono state monitorate sino a maggio 2014 [12, 13] per presentazioni o comunicazioni di dati aggiuntivi concernenti l'analisi della letteratura.

La ricerca ha riguardato gli articoli in lingua inglese o italiana, pubblicati tra il 2009 ed il 2014 in riferimento allo specifico contesto italiano, ed è stata articolata considerando 2 grandi raggruppamenti tematici: il primo epidemiologico, con l'obiettivo di estrarre dati epidemiologici relativi alle patologie HCV-indotte; il secondo economico, con lo scopo di indagare sui dati di costo gravanti sul SSN e sulla società.

Il processo sistematico è stato articolato in 4 fasi: identificazione, screening, eleggibilità e inclusione, (in accordo con quanto riportato nelle linee guida raccomandate per l'analisi sistematica della letteratura scientifica [14]). I search terms utilizzati per l'estrazione degli articoli epidemiologici dai database elettronici sono stati:

- Pubmed: ((Hepacivirus [MeSH Terms]) OR (Hepatitis C, Chronic/pathology [MeSH Terms])) AND (Italy [Title/Abstract])

- EPICENTRO: HCV OR Epatite C

- PharmacoEcomomics: Epatite_C AND HCV AND NOT (B AND A AND HBV)

- Farmeconomia: HCV

- Il sole 24 ore Sanità: HCV OR Epatite C

Search terms utilizzati per l'estrazione degli articoli economici dai database elettronici:

- Pubmed: ((((cost of illness [MeSH Terms]) OR cost [MeSH Terms]) OR burden of disease [MeSH Terms]) OR burden of illness [MeSH Terms]) AND ((Hepacivirus [MeSH Terms]) OR (HCV[Title]) OR (hepatitis $\mathrm{C}$ virus infection [Title]) OR (Pathologies induced by HCV [Title]) OR (Hepatitis C Pathologies)) AND (Italy [Affiliation])

\footnotetext{
${ }^{1}$ Associazione Italiana per lo Studio del Fegato.

${ }^{2}$ Sistema Informativo Trapianti.
}

- EPICENTRO: HCV OR Epatite C AND costo OR spesa

- PharmacoEcomomics: (costo OR spesa OR) AND (hcv)

- Farmeconomia: HCV

- Il sole 24 ore Sanità: spesa OR costo AND hcv OR epatite C

Per l'inclusione, 2 ricercatori hanno indipendentemente rivisto gli studi, utilizzando il titolo, l'abstract o il testo in esteso. Le discrepanze sono state discusse e risolte mediante analisi e confronto con altri esperti. Tutti gli studi utilizzati per la determinazione dei parametri epidemiologici ed economici dovevano soddisfare almeno uno dei seguenti criteri di inclusione:

1. fare riferimento a dati epidemiologici (casi incidenti di malattia, dati di prevalenza o probabilità di transizione) derivati da database di popolazione, indagini nazionali o da registri;

2. fare riferimento a dati di costo diretti, valutati nella prospettiva del SSN, ed espressi in valori monetari per gruppi ospedalieri correlati alla malattia (DRG) e tariffe ambulatoriali;

3. fare riferimento a studi osservazionali concernenti dati epidemiologici sulle malattie indotte da HCV ed i loro costi diretti e/o indiretti associati, registrati contemporaneamente nella stessa coorte di pazienti;

4. fare riferimento a dati italiani rilevanti per $\mathrm{HCV}$ e le patologie $\mathrm{HCV}$-indotte.

Altri studi o valutazioni economiche ed epidemiologiche che non rispondevano ai criteri di inclusione sopra menzionati sono stati esclusi. Al termine del processo revisione sistematica sono stati identificati 18 articoli per il raggruppamento tematico epidemiologico e 12 per il raggruppamento tematico economico riguardanti le patologie $\mathrm{HCV}$-indotte (Fig. 1).

\subsection{Parametri epidemiologici}

I principali parametri epidemiologici utilizzati per informare il modello di COI sono riassunti in Tabella 1. I diversi possibili stati di malattia sono coerenti con quelli considerati da altri autori $[7,8]$. In Italia, la popolazione residente al 1 gennaio 2013 ammontava a circa 60 milioni (59.685.227) [16]. Il modello ha assunto una stima della prevalenza di $\mathrm{HCV}$ in Italia tra il $2 \%$ e il $3,5 \%$ [5, 17]. Il virus dell' $\mathrm{HCV}$ presenta differenti varianti genetiche che possono essere incluse in tre gruppi principali: il genotipo 1 che si riscontra frequentemente nella popolazione italiana, i genotipi $2 / 3$ e il genotipo 4+altri [8, 18]. Genericamente, l'infezione da HCV evidenzia un elevato tasso di cronicizzazione, che può variare tra il 50\% e l'85\% [19]. Nella maggior parte dei casi, quando un soggetto è esposto al virus, l'infezione evolve in epatite cronica in modo del tutto silente (80\% dei casi) [8]. 


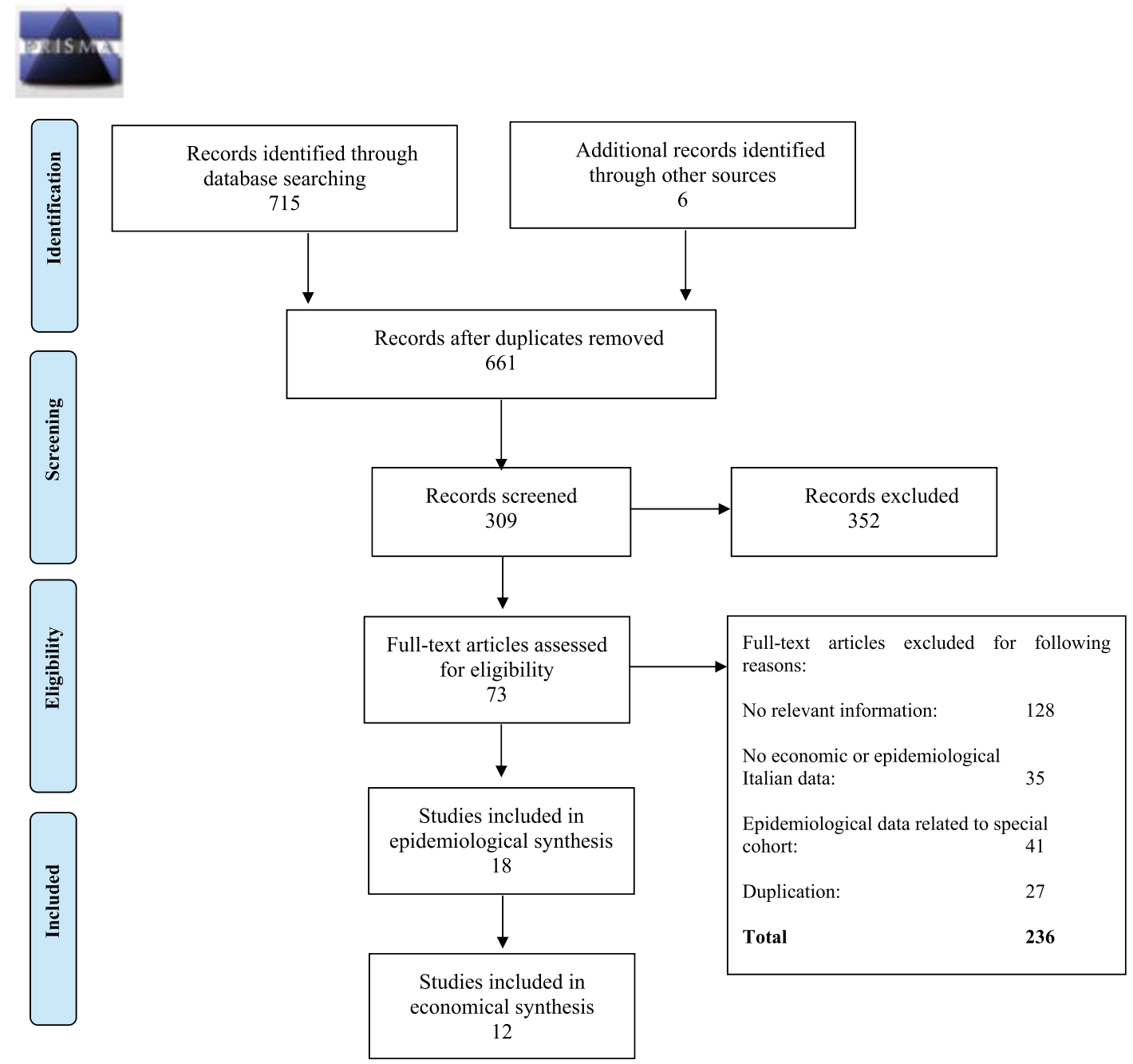

Fig. 1 PRISMA 2009 Flow Diagram. (From: Moher D., Liberati A., Tetzlaff J., Altman D.G., The PRISMA Group (2009). Preferred Reporting Items for Systematic Reviews and Meta-Analyses: The PRISMA Statement. PLoS Med 6(6): e1000097. doi:10.1371/journal.pmed1000097)

Data l'asintomaticità della patologia, i pazienti diagnosticati e gestiti da centri clinici rappresentano solo una parte esigua (4\%-8\%) dell'intera popolazione affetta da epatite cronica $\mathrm{C}[8,20]$. Attualmente, si ritiene che una percentuale variabile tra il $19,5 \%$ e il $33,1 \%$ dei pazienti in cui sia stata diagnosticata una infezione cronica da HCV sia sottoposta a trattamento antivirale [21, 22].

\section{Parametri di costo}

\subsection{I costi diretti sanitari}

Tramite la revisione sistematica della letteratura scientifica disponibile sono stati individuati i dati di costo diretto associati a ciascuno stato di malattia. I costi sono stati successivamente attualizzati al 2013 mediante procedura di riparametrizzazione per confronto con l'Indice dei prezzi per le rivalutazioni monetarie ISTAT [23].
I dati fanno riferimento a costi annui aggregati relativi al management delle malattie HCV-indotte: visite specialistiche, analisi ematologiche e sierologiche, procedure diagnostiche di controllo, terapie farmacologiche di supporto ed ospedalizzazioni [4, 5]. I dettagli relativi ai parametri ed ai range probabilistici considerati nel modello sono sintetizzati in Tabella 1.

Per i pazienti con infezione cronica e cirrosi compensata sono stati considerati anche i costi dei trattamenti con duplice (Interferone Pegilato (Peg-IFN) e Ribavirina [5]) e triplice terapia (Peg-IFN, Ribavirina Telaprevir/Boceprevir), considerando il dosaggio medio riportato in scheda tecnica ed il prezzo per il SSN al netto degli sconti previsti per legge [8]. I pazienti affetti da epatocarcinoma assorbono un range di spesa compreso tra $€ 4.827$ e $€ 6.786$ per anno [29, 31]. I costi dei trapianti variano tra $€ 73.763$ e $€ 90.162$ per l'intervento $[32,33]$ e tra $€ 3.867$ e $€ 5.800$ per il trattamento attuato nel primo anno successivo all'intervento [31, 32]. 
Tabella 1 Parametri epidemiologici e parametri per costi diretti sanitari associati agli stati di salute $\mathrm{HCV}$-correlati

\begin{tabular}{|c|c|c|}
\hline Parametri epidemiologici & Range (Min-Max) & Fonte \\
\hline Popolazione residente & 59.685 .227 & {$[16]$} \\
\hline Prevalenza HCV & $(2 \%-3,5 \%)$ & {$[5,17]$} \\
\hline Percentuale pz G1 & $(52,40 \%-56,00 \%)$ & {$[8,18]$} \\
\hline Percentuale pz G2/G3 & $(36,40 \%-40,00 \%)$ & {$[8,18]$} \\
\hline Percentuale pz G4 e altri & $(5,00 \%-11,20 \%)$ & {$[8,18]$} \\
\hline Frequenza cronicizzazione & $(50 \%-85 \%)$ & {$[19]$} \\
\hline$\%$ pazienti gestiti dai centri clinici & $(4 \%-8 \%)$ & {$[8,20]$} \\
\hline$\%$ pazienti gestiti dai centri clinici e trattati & $(19,5 \%-33,1 \%)$ & {$[21,22]$} \\
\hline Incidenza HCV & $(0,013 \%-0,025 \%)$ & {$[24]$} \\
\hline Cirrosi & $(13,40 \%-26,20 \%)$ & {$[24,25]$} \\
\hline$\%$ pazienti trattati con cirrosi (su totale trattati) & $(6,7 \%-53,50 \%)$ & {$[22,26]$} \\
\hline$\%$ trattati con cirrosi compensata & $(81,7 \%-89,0 \%)$ & {$[21,26]$} \\
\hline$\%$ trattati con cirrosi scompensata & $(11,0 \%-19,3 \%)$ & {$[21,26]$} \\
\hline$\%$ in osservazione con cirrosi compensata & $90 \%$ & {$[21]$} \\
\hline$\%$ in osservazione con cirrosi scompensata & $10 \%$ & {$[21]$} \\
\hline Carcinomi su cirrotici compensati & $(0,5 \%-7,2 \%)$ & {$[26,27]$} \\
\hline Carcinomi su cirrotici scompensati & $(5,14 \%-8,46 \%)$ & [28] \\
\hline$\%$ trapianti HCV-correlati (sul totale dei gestiti) & $0,76 \%$ & {$[12,13]$} \\
\hline Tasso di mortalità cirrosi compensata & $(1,50 \%-5,00 \%)$ & {$[21]$} \\
\hline Tasso di mortalità cirrosi scompensata & $(6,10 \%-18,30 \%)$ & {$[28]$} \\
\hline Tasso di mortalità carcinoma & $(19,4 \%-42,70 \%)$ & [29] \\
\hline Tasso di mortalità trapianto (1 anno) & $(6,00 \%-42,00 \%)$ & {$[30]$} \\
\hline Costi diretti & Range (Min-Max) & Fonte \\
\hline Epatite cronica & $(€ 232-€ 354)$ & {$[31,32]$} \\
\hline Cirrosi compensate & $(€ 299-€ 500)$ & {$[29,31]$} \\
\hline Cirrosi scompensata & $(€ 3.535-€ 5.264)$ & {$[29,31]$} \\
\hline Carcinoma & $(€ 4.827-€ 6.786)$ & {$[29,31]$} \\
\hline Trapianto (intervento) & $(€ 73.763-€ 90.162)$ & {$[32,33]$} \\
\hline Trapianto (trattamento $1^{\circ}$ anno) & $(€ 3.867-€ 5.800)$ & {$[31,32]$} \\
\hline
\end{tabular}

\subsection{I costi indiretti}

I parametri per la stima dei costi indiretti sono sintetizzati in Tabella 2. Per ogni cittadino italiano è stato stimato il salario medio annuo corrispondente a $€ 28.539$ [34], equivalente in media ad un compenso/die pari a $€ 121,26$ al lordo delle tasse; il valore minimo è dato dal reddito medio annuo suddiviso per i giorni lavorativi dell'anno 2013 (pari a 253 giorni effettivi) [35]. Viceversa, il valore massimo del reddito medio giornaliero è stato ottenuto dividendo il reddito annuo per giorni lavorativi escludendo le ferie (pari a 220 giorni) [36]. Il numero delle giornate lavorative perse è stato stimato in maniera conservativa sulla base dei dati pubblicati nel Libro Bianco AISF 2011 [4] e considerando il valore delle assenze dal lavoro accordate ai pazienti che beneficiano della legge 104, relativa alle malattie croniche invalidanti o neoplastiche [37]. Per la stima dei costi indiretti e per quel- li associati alla mortalità, è stato assunto conservativamente che solo una quota parte dei soggetti fosse in età produttiva ed occupata [19, 21, 22, 38].

\subsection{Analisi statistica}

Per considerare la variabilità intrinseca dei dati impiegati per informare il modello, è stato utilizzato un approccio probabilistico [Probabilistic Sensitivity Analysis (PSA)]. L'analisi consiste nell'utilizzare le differenze trovate nelle diverse fonti esaminate come indicative di minimo e massimo della distribuzione di incertezza di ogni parametro considerato.

La scelta della distribuzione probabilistica è stata attribuita applicando quanto generalmente riportato per lo sviluppo dei modelli probabilistici nelle valutazioni economiche, distinguendo tra costi (distribuzione gamma) e parametri epidemiologici (distribuzione beta) [15]. Inoltre, le distri- 
Tabella 2 Parametri per costi indiretti associati agli stati di salute HCV-correlati

\begin{tabular}{llllll}
\hline Costi indiretti & $\begin{array}{l}\text { Giorni lavorativi } \\
\text { persi }[6,37]\end{array}$ & Costo/die & Min [34,35] & Max [34,36] & $\begin{array}{l}\text { \% pazienti occupata } \\
{[19,21,22,38]}\end{array}$ \\
\hline Pazienti con epatite cronica trattati & 50 & $€ 121,26$ & $€ 112,80$ & $€ 129,72$ & $75 \%$ \\
Pazienti con epatite cronica non trattati & 18 & $€ 121,26$ & $€ 112,80$ & $€ 129,72$ & $55 \%$ \\
Pazienti con cirrosi trattati & 70 & $€ 121,26$ & $€ 112,80$ & $€ 129,72$ & $75 \%$ \\
Pazienti con cirrosi non trattati & 21 & $€ 121,26$ & $€ 112,80$ & $€ 129,72$ & $55 \%$ \\
Pazienti con epatocarcinoma & 90 & $€ 121,26$ & $€ 112,80$ & $€ 129,72$ & $50 \%$ \\
Pazienti con trapianto di fegato & 180 & $€ 121,26$ & $€ 112,80$ & $€ 129,72$ & $50 \%$ \\
Decesso per cause correlate all'HCV & 220 & $€ 121,26$ & $€ 112,80$ & $€ 129,72$ & $50 \%$ \\
\hline
\end{tabular}

Tabella 3 Numero di pazienti in carico al SSN per stato patologico e terapia per anno

\begin{tabular}{|c|c|c|c|c|}
\hline \multirow[t]{2}{*}{ Stato di malattia } & \multicolumn{2}{|l|}{ Trattati } & \multirow{2}{*}{$\begin{array}{l}\text { Osservazione/ } \\
\text { terapia supporto } \\
\text { (Min-Max) }\end{array}$} & \multirow{2}{*}{$\begin{array}{l}\text { Totale pz } \\
\text { (Min-Max) }\end{array}$} \\
\hline & $\begin{array}{l}\text { Duplice } \\
\text { (Min-Max) }\end{array}$ & $\begin{array}{l}\text { Triplice } \\
\text { (Min-Max) }\end{array}$ & & \\
\hline $\mathrm{HCV}$ cronica & $\begin{array}{l}13.965 \\
(7.215-22.909)\end{array}$ & $\begin{array}{l}796 \\
(410-1.309)\end{array}$ & $\begin{array}{l}50.971 \\
(28.616-79.673)\end{array}$ & $\begin{array}{l}65.732 \\
(36.241-103.891)\end{array}$ \\
\hline Cirrosi compensata & $\begin{array}{l}3.692 \\
(253-11.613)\end{array}$ & $\begin{array}{l}210 \\
(14-661)\end{array}$ & $\begin{array}{l}202.491 \\
(112.422-318.623)\end{array}$ & $\begin{array}{l}206.393 \\
(112.689-330.897)\end{array}$ \\
\hline Cirrosi scompensata & \multicolumn{2}{|c|}{$\begin{array}{l}662 \\
(40-2.131)\end{array}$} & $\begin{array}{l}21.282 \\
(11.834-33.455)\end{array}$ & $\begin{array}{l}21.944 \\
(11.874-35.586)\end{array}$ \\
\hline Carcinoma & \multicolumn{2}{|c|}{4.622} & - & $\begin{array}{l}4.622 \\
(4-23.294)\end{array}$ \\
\hline Trapianti & $\begin{array}{l}50 \\
(28\end{array}$ & $(285-783)$ & - & $\begin{array}{l}504 \\
(285-783)\end{array}$ \\
\hline Totale & \multicolumn{2}{|c|}{$\begin{array}{l}24.451 \\
(8.221-62.700)\end{array}$} & $\begin{array}{l}274.744 \\
(152.872-431.751)\end{array}$ & $\begin{array}{l}299.195 \\
(161.093-494.451)\end{array}$ \\
\hline
\end{tabular}

buzioni di ogni parametro sono state utilizzate per effettuare 5.000 simulazioni Monte Carlo in modo da ottenere stime intervallari [Intervallo di Confidenza (IC) al 95\%] dei principali dati epidemiologici ed economici.

Infine, per verificare l'incertezza del modello è stata condotta un'analisi di sensibilità ad una via, facendo variare ogni parametro per i valori più alti e più bassi possibile del dato ottenuto nella revisione sistematica della letteratura. Come valore medio della distribuzione, è stato inserito il dato proveniente da una fonte di letteratura particolarmente attendibile, o la media tra minimo e massimo, assumendo così una equidistribuzione dei valori intorno ai due estremi. Questo tipo di analisi permette di identificare quali parametri influenzano maggiormente la variazione dei costi finali associati alle patologie $\mathrm{HCV}$-indotte. La definizione di possibile per i valori più alti e più bassi derivanti dall'analisi della letteratura può variare da modello a modello, ma solitamente risulta ragionevole variare i parametri in base agli intervalli di confidenza dei dati.

\section{Risultati}

Il modello epidemiologico ha consentito di stimare il numero dei pazienti prevalenti con diagnosi di patologia $\mathrm{HCV}$ indotta in Italia, in un anno. Tale stima risulta corrispondere a 299.195 (IC95\%: 161.093-494.451) soggetti.

Non tutti i pazienti diagnosticati soddisfano i criteri per l'istituzione di un determinato trattamento farmacologico e la maggior parte resta in osservazione, effettuando controlli periodici per la verifica dello stato di patologia [19]. Ne consegue che i pazienti trattati con cure specifiche ammontano a 24.451 (IC95\%: 8.221-62.700), ossia l' $8,2 \%$ dei soggetti diagnosticati in carico al SSN. I trattamenti per l'infezione cronica e la cirrosi compensata (i.e. duplice e triplice terapia) interessano il 76,3\% dell'intera popolazione di trattati. Di questi, la quota parte trattata con duplice terapia corrisponde al 94,6\% (Tabella 3).

I dati di costo diretto sanitario ed indiretti, stimati per singolo paziente italiano nel 2013, sono riportati in Fig. 2. Nello specifico e al netto dei costi per duplice e triplice tera- 


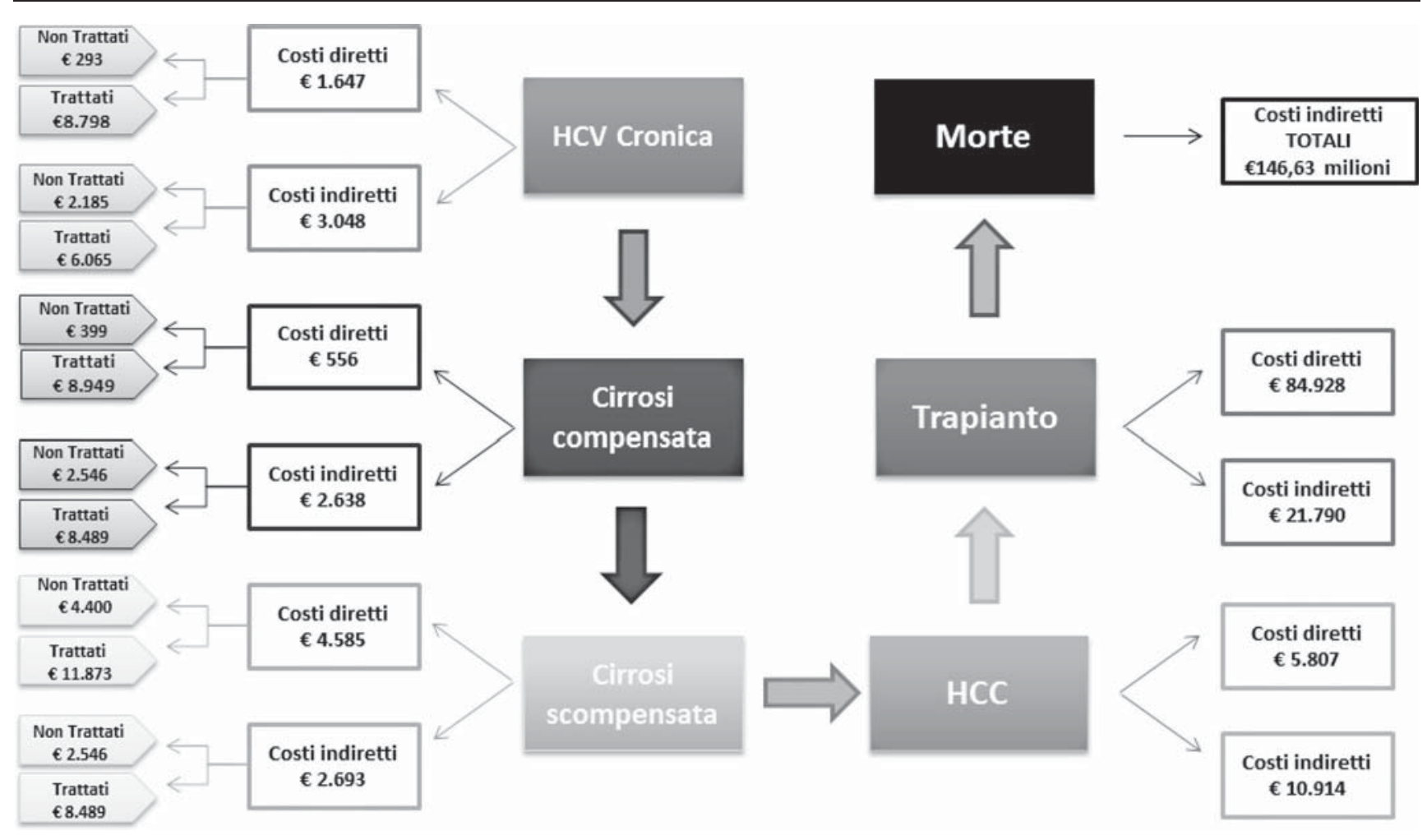

Fig. 2 Costo medio annuo per paziente affetto da patologie HCV-indotte

pia, un paziente con $\mathrm{HCV}$ cronica comporta una spesa media pari a $€ 1.647$ all'anno, $€ 556$ se è affetto da cirrosi compensata, €4.585 se la cirrosi è scompensata, €5.807 se è colpito da $\mathrm{HCC}$ e $€ 84.928$ se subisce un trapianto.

In riferimento ai costi indiretti, il modello ha stimato una perdita di produttività pari $\mathrm{a} € 3.048$ per i pazienti con $\mathrm{HCV}$ cronica, $€ 2.638$ per quelli con cirrosi, $€ 10.914$ nel caso di epatocarcinoma e $€ 21.790$ per coloro che sono sottoposti a trapianto di fegato. Per la stima dei costi indiretti, è opportuno tener conto anche della perdita di produttività attribuibile al decesso, che genera un onere indiretto massimo per la società complessivamente pari a 146,62 milioni (IC95\%: $€ 60,57-€ 270,06$ milioni) di euro per anno (Tabella 4).

Complessivamente, la spesa annua in Italia risulta pari a $€ 1,05$ miliardo (IC95\%: $€ 0,609-€ 1,6$ miliardi), di cui il 38,6\% (€407 milioni, IC95\%: €231-€633 milioni) è assorbito da costi diretti sanitari, mentre la perdita di produttività associata all'assenza dal lavoro supera i €645 milioni (IC95\%: €369-€996 milioni) e rappresenta il 61,3\% dell'onere totale sostenuto dalla società. Nella Tabella 4 può essere osservata nel dettaglio la ripartizione dei costi per patologia.

La Fig. 3 mostra i risultati dell'analisi di sensitività oneway. La prevalenza dell'HCV risulta essere il principale parametro epidemiologico capace di generare il livello di variazione più elevato nella stima dei costi diretti (Fig. 3a). Infatti, l'oscillazione di tale parametro compresa tra il 2,0\% e il $3 \%$ comporta variazioni elevate della spesa diretta sanitaria $( \pm 27,3 \%)$. La frequenza di cronicizzazione è il secondo parametro più rilevante: ipotizzando che questa assuma il valore massimo di $85 \%$, l'aumento dei costi totali è del $+26,9 \%$ circa; se si ipotizza che il parametro assuma il valore minimo del $50 \%$, il decremento dei costi totali risulta similarmente speculare $(-26,9 \%)$. La variazione dei costi ha effetti minori sul modello. In effetti, il costo della cirrosi compensata e quello della cirrosi scompensata producono una variazione sulla stima dei costi totali pari rispettivamente a $\pm 4,9 \%$ e $\pm 4,1 \%$. La Fig. $3 b$ mostra i risultati dell'analisi di sensibilità ad una via per costi indiretti. Ancora una volta la prevalenza è il parametro che più influenza i costi $( \pm 27,2 \%)$. Una variazione minore è attribuibile al tasso di mortalità per cirrosi compensata $( \pm 7,49 \%)$ e al costo di un giorno lavorativo $( \pm 6,98 \%)$.

\section{Discussione}

L'infezione cronica da $\mathrm{HCV}$ è una condizione patologica ad elevato impatto economico e sociale in tutto il mondo, ed in particolare in Italia, dove la prevalenza si attesta attorno al 2,0-3,5\% [8]. Ad oggi, non esiste uno studio di prevalenza dell'infezione da HCV su un campione rappresentativo dell'intera popolazione Italiana [4]. Per questo motivo, 
Tabella 4 Onere complessivo delle patologie $\mathrm{HCV}$-indotte. Costi diretti ed indiretti distinti per condizione patologica $(€$ in milioni $)$

\begin{tabular}{|c|c|c|c|}
\hline Stato di malattia & $\begin{array}{l}\text { Costi diretti } \\
\text { (Min-Max) }\end{array}$ & $\begin{array}{l}\text { Costi indiretti } \\
\text { (Min-Max) }\end{array}$ & $\begin{array}{l}\text { Costi totali } \\
\text { (Min-Max) }\end{array}$ \\
\hline $\mathrm{HCV}$ cronica & $\begin{array}{l}€ 126,92 \\
(€ 65,75-€ 204,69)\end{array}$ & $\begin{array}{l}€ 127,86 \\
(€ 71,20-€ 200,82)\end{array}$ & $\begin{array}{l}€ 254,78 \\
(€ 136,19-€ 403,46)\end{array}$ \\
\hline Cirrosi & $\begin{array}{l}€ 211,84 \\
(€ 113,26-€ 340,78)\end{array}$ & $\begin{array}{l}€ 340,60 \\
(€ 192,88-€ 529,63)\end{array}$ & $\begin{array}{l}€ 552,45 \\
(€ 303,22-€ 863,55)\end{array}$ \\
\hline Carcinoma & $\begin{array}{l}€ 26,37 \\
(€ 0,03-€ 131,19)\end{array}$ & $\begin{array}{l}€ 24,72 \\
(€ 0,03-€ 122,24)\end{array}$ & $\begin{array}{l}€ 51,09 \\
(€ 0,043-€ 260,91)\end{array}$ \\
\hline Trapianti & $\begin{array}{l}€ 42,71 \\
(€ 23,87-€ 66,94)\end{array}$ & $\begin{array}{l}€ 5,48 \\
(€ 3,04-€ 8,63)\end{array}$ & $\begin{array}{l}€ 48,19 \\
(€ 26,69-€ 75,52)\end{array}$ \\
\hline Morte & - & $\begin{array}{l}€ 146,62 \\
(€ 60,57-€ 270,06)\end{array}$ & $\begin{array}{l}€ 146,62 \\
(€ 60,57-€ 270,06)\end{array}$ \\
\hline Totale & $\begin{array}{l}€ 407,85 \\
(€ 231,51-€ 633,28)\end{array}$ & $\begin{array}{l}€ 645,30 \\
(€ 369,59-€ 992,13)\end{array}$ & $\begin{array}{l}€ 1.053,14 \\
(€ 609,45-€ 1.616,19)\end{array}$ \\
\hline
\end{tabular}

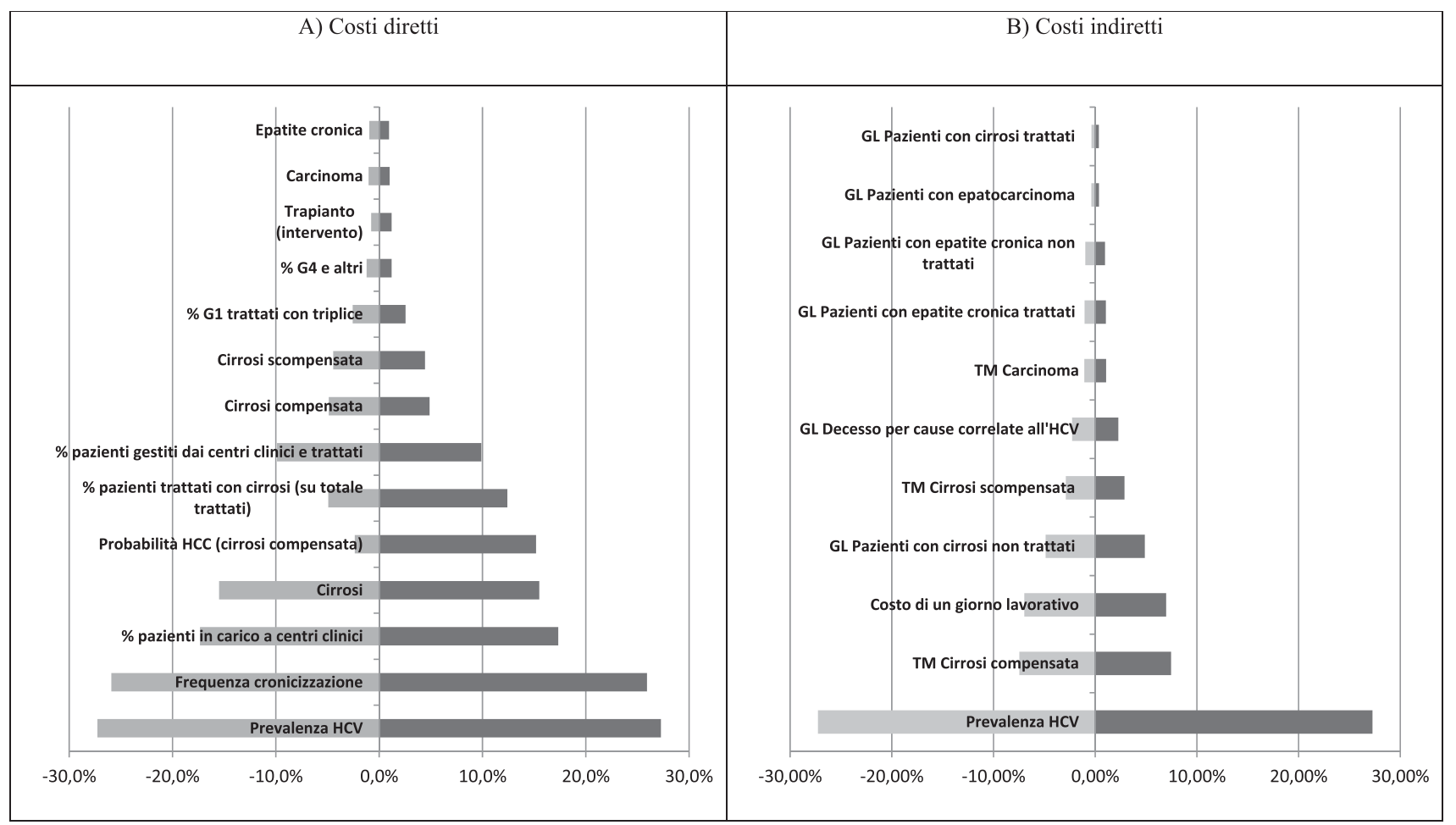

Fig. 3 Analisi di sensibilità one-way-Grafico tornado

è stato necessario ricostruire la stima dei principali indicatori epidemiologici tramite alcuni studi europei [5, 17], che evidenziano come la situazione italiana sia peculiare. Infatti, nel nostro paese i tassi di prevalenza sono tra i più alti in Europa.

Il modello epidemiologico ha consentito di stabilire che il numero di pazienti prevalenti con diagnosi di patologie $\mathrm{HCV}$-indotte nel nostro paese dovrebbe corrispondere a 299.195 individui. Inoltre, lo studio ha permesso di calcolare l'onere economico assorbito dalle patologie $\mathrm{HCV}$-indotte nel 2013, stimando un costo medio annuo di poco superiore ad 1,0 miliardo di Euro.

Esaminando i risultati di spesa per patologia HCVindotta, si può osservare che gli stati patologici avanzati (i.e. cirrosi compensata, cirrosi scompensata, epatocarcinoma) determinano un elevato assorbimento di risorse economiche. La spesa raggiunge valori particolarmente elevati quando sopraggiunge la necessità di intervenire con un trapianto di fegato. Infatti, sebbene i pazienti sottoposti a trapianto siano una quota esigua (meno dello $0,2 \%$ dell'intera popola- 
zione con diagnosi di patologia $\mathrm{HCV}$-indotta), la spesa che si genera risulta pari al $10,5 \%$ della spesa sostenuta per costi diretti sanitari dal SSN e al 4,6\% della spesa totale calcolata includendo anche i costi indiretti.

I costi diretti sanitari sostenuti dal SSN in Italia, nel 2013, per le patologie HVC-indotte sono inclusi tra i €231,51 e $€ 633,28$ milioni. Tali valori sono linea con quanto riportato in uno studio recentemente pubblicato per il contesto nazionale [8], in cui la spesa diretta sanitaria risultava compresa tra $\mathrm{i} € 388$ e i $€ 666$ milioni di Euro. Tuttavia, il lavoro di Mennini et al. non considerava i costi indiretti e non aveva come obiettivo specifico quello di determinare i costi stratificati per patologia $\mathrm{HCV}$-indotta. La possibilità di condurre ulteriori confronti con altre pubblicazioni è limitata. In effetti, alcuni studi internazionali hanno stimato il burden causato dall'HCV in diversi paesi europei ed extra-europei, ma nessuno di questi studi includeva anche la prospettiva italiana [39, 40].

Il modello sviluppato evidenzia che i costi indiretti superano i €645 milioni (IC95\%: €369,59-€992,13), gravando per circa il $61 \%$ sui costi totali.

La PSA ha confermato l'importanza di attuare misure di prevenzione e di intervento precoce nella gestione dei pazienti con HCV. Infatti, per ridurre visibilmente l'impatto economico delle patologie $\mathrm{HCV}$-indotte è fondamentale impedire o limitare la diffusione del virus e/o che l'infezione si cronicizzi.

Questo studio presenta alcuni limiti. In primo luogo va sottolineato che il modello è basato su dati campionari o rilevati in una sola regione o in singole compagini territoriali. La causa risiede nell'impossibilità di individuare un ente nazionale unico che registri tutti i dati di costo ed epidemiologici riferiti alla malattia. Tuttavia, una revisione sistematica della letteratura, rigorosamente condotta seguendo precise linee guida internazionali [14], ha permesso di identificare le fonti più recenti e comunemente riconosciute dalla comunità scientifica nazionale. Inoltre, le analisi di sensibilità deterministica e probabilistica hanno consentito di tenere in dovuta considerazione l'eterogeneità dei differenti dati disponibili, ottenendo stime intervallari includenti l'incertezza complessiva delle fonti utilizzate.

In secondo luogo, a causa di scarse o mancanti informazioni, non è stato possibile stimare alcune voci di spesa associate alla malattia e, dunque, i risultati presentati tendono a sottostimare l'onere economico complessivo realmente causato dall'HCV. In particolare nella stima dei costi indiretti, è stata considerata solo la perdita di produttività attribuita alle patologie HCV-indotte. Tuttavia, una valutazione di Cost of Illness dovrebbe prevedere la stima completa dei costi indiretti reali, comprendendo anche la perdita di produttività e di competitività associate al presenteismo (presenza al lavoro con ridotta quantità e qualità produttiva). Inoltre, $\mathrm{i}$ costi diretti sanitari sono stati calcolati sulla base di tariffe aggregate basate sul sistema DRG, che include gli interventi chirurgici, i materiali e il personale per ogni singola diagnosi e la tariffa di rimborso corrispondente alla somma di tutte le prestazioni erogate [9]. Infine, nella presente valutazione non si è tenuto conto dei costi sostenuti direttamente dai pazienti (costi out-of-pocket). Tuttavia, per quanto a nostra conoscenza, questo risulta essere il primo studio che ha considerato sia i costi diretti (sostenuti dal SSN) che quelli indiretti (a carico della societá) per stimare l'onere complessivo associato alle patologie $\mathrm{HCV}$-indotte nel nostro paese. In conclusione, il presente lavoro può essere ritenuto un' efficiente strumento di riferimento per i decisori pubblici che vogliano comprendere adeguatamente gli aspetti economici generati dalla gestione e dal trattamento delle patologie $\mathrm{HCV}$-indotte in Italia.

\section{Conflitti di interesse Nessuno.}

\section{Bibliografia}

1. Shepherd J, Jones J, Hartwell D, Davidson P, Price A, Waugh $\mathrm{N}$. Interferon alfa (pegylated and non-pegylated) and ribavirin for the treatment of mild chronic hepatitis $\mathrm{C}$ : a systematic review and economic evaluation. Health Technology Assessment. 2007;11(11):1-205.

2. Ciccozzi M, Lo Presti A, Ciccaglione AR, Zehender G, Ciotti M. Phylogeny and phylodinamic of Hepatitis C in Italy. BMC Infect Dis. 2012;12(Suppl 2):S5.

3. Oleari F. L'impegno istituzionale nella lotta contro le epatiti: entro due anni un Piano nazionale con fondi dedicati. Il sole 24 ore Sanità, I quaderni di medicina. Giugno 2013. p. 28-30.

4. Libro Bianco AISF 2011. Proposta per un piano nazionale per il controllo delle malattie epatiche. Definizione ambiti e possibili interventi. 2011. http://www.webaisf.org/media/13891/ libro-bianco-aisf-2011.pdf (ultimo accesso Marzo 2014).

5. European Centre for Disease Prevention and Control. Annual Epidemiological Report 2013. Reporting on 2011 surveillance data and 2012 epidemic intelligence data. Stockholm: ECDC; 2013.

6. Fagiuoli S, Lucà MG, Magini G, De Giorgio M. Puntare sulla prevenzione delle epatiti come strategia per evitare che vi sia progressione dell'epatocarcinoma. Il sole 24 ore Sanità, I quaderni di medicina. Giugno 2013. p. 22-27.

7. Cammà C, Sacchini D, Gasbarrini A. Percorsi diagnosticoterapeutici in pazienti con epatite cronica da HCV genotipo 1: chi e come esegue il trattamento. Il sole 24 ore Sanità, I quaderni di medicina. Giugno 2013. p. 12-15.

8. Mennini FS, Marcellusi A, Andreoni M, Gasbarrini A, Salomone $\mathrm{S}$, Craxì A. Health policy model: long-term predictive results associated with the management of hepatitis $\mathrm{C}$ virus-induced diseases in Italy. ClinicoEcon Outcomes Res. 2014;6:303-10.

9. Baio G, Capone A, Marcellusi A, Mennini FS, Favato G. Economic burden of human papillomavirus-related diseases in Italy. PLoS ONE. 2012;7(11):e49699. doi:10.1371/journal. pone.0049699.

10. Marcellusi A, Viti R, Mecozzi A, Mennini FS. Direct and Indirect Cost of Diabetes in Italy: A Prevalence Probabilistic Approach. CEIS Working Paper No. 321. Roma, 2014. http://papers. ssrn.com/sol3/papers.cfm?abstract_id=2476429 (ultimo accesso agosto 2014). 
11. Joel E, Segel BA. Cost-of-Illness Studies-A Primer. RTI International RTI-UNC Center of Excellence in Health Promotion Economics. Gennaio 2006. https://www.rti.org/pubs/coi_primer.pdf (ultimo accesso marzo 2014).

12. Associazione Italiana per lo Studio del Fegato. Trapianto di fegato non urgente nell' adulto. http://www.webaisf.org/media/8065/ documento_trapianto.pdf (ultimo accesso maggio 2014).

13. Ministero della Salute. Sistema Informativo Trapianti. https:// trapianti.sanita.it/statistiche/PEorg.asp (ultimo accesso maggio 2014).

14. Moher D, Liberati A, Tetzlaff J, Altman DG, The PRISMA Group. Preferred reporting items for systematic reviews and meta-analyses: the PRISMA statement. PLoS Med. 2009;6(6):e1000097. doi:10.1371/journal.pmed1000097.

15. Briggs A, Claxton K, Sculpher M. Decision modelling for health economic evaluation. New York: Oxford University Press Inc.; 2007.

16. Popolazione residente al 1 Gennaio 2013 per età, sesso e stato civile Italia. http://demo.istat.it/pop2013/index.html (ultimo accesso Marzo 2014).

17. Esteban JI, Sauleda S, Quer J. The changing epidemiology of hepatitis C virus infection in Europe. J Hepatol. 2008;48:148-62.

18. Marascio N, Matera G, Quirino A, Giancotti A, Barreca GS, Lamberti AG, Caroleo B, Liberto MC, Focà A. Eleven-year distribution pattern of hepatitis $\mathrm{C}$ virus in Southern Italy. J Pathogens. 2012;2012:631095. 7 pp. doi:10.1155/2012/631095.

19. AISF. Practice guidelines for the treatment of hepatitis C: recommendations from an AISF/SIMIT/SIMAST Expert Opinion Meeting. Dig Liver Dis. 2010;42:81-91.

20. Maratea D, Messori A, Fadda V. Nationwide prediction of future expenditure for protease inhibitors in chronic hepatitis C. Dig Liver Dis. 2012;44:85-7.

21. Mariano A, Caserta C, Pendino GM, Vacalebre C, Surace P, Fiorillo MT, Polito I, Surace M, Alicante S, Amante A, et al. Antiviral treatment for hepatitis $\mathrm{C}$ virus infection: effectiveness at general population level in a highly endemic area. Dig Liver Dis. 2009;41:509-15.

22. Stroffolini T, Spadaro A, Guadagnino V, et al. Current practice of hepatitis C treatment in Southern Italy. Dig Liver Dis. 2010;42:822-5.

23. ISTAT. Indice dei prezzi al consumo per le rivalutazioni monetarie. Available: http://rivaluta.istat.it/ (ultimo accesso ottobre 2013).

24. Mariano A, Scalia Tomba G, Tosti ME, Spada E, Mele A. Estimating the incidence, prevalence and clinical burden of hepatitis $\mathrm{C}$ over time in Italy. Scand J Infect Dis. 2009;41:689-99.

25. Sagnelli E, Stroffolini T, Mele A, Almasio PL, Coppola N, Ferrigno $\mathrm{L}$, et al. The importance of $\mathrm{HCV}$ on the burden of chronic liver disease in Italy: a multicenter prevalence study of 9,997 cases. J Med Virol. 2005;75:522-7.

26. Fattovich G, Giustina G, Degos F, Tremolada F, Diodati G, Almasio P, Nevens F, Solinas A, Mura D, Brouwer JT, Thomas
H, Njapoum C, Casarin C, Bonetti P, Fuschi P, Basho J, Tocco A, Bhalla A, Galassini R, Noventa F, Schalm SW, Realdi G. Morbidity and mortality in compensated cirrhosis type C: a retrospective follow-up study of 384 patients. Gastroenterology. 1997;112(2):463-72.

27. Deuffic-Burban S, Deltenre P, Buti M, et al. Predicted effects of treatment for $\mathrm{HCV}$ infection vary among European countries. Gastoenterology. 2012;143:974-85.

28. Planas R, Ballesté B, Alvarez MA, Rivera M, Montoliu S, Galeras JA, Santos J, Coll S, Morillas RM, Solà R. Natural history of decompensated hepatitis $C$ virus-related cirrhosis. A study of 200 patients. J Hepatol. 2004;40(5):823-30.

29. Cicchetti A, Ruggeri M, Coretti S, Piscaglia A, Ponziani FR, Lanati E, Gasbarrini A. Valutazione economica di un programma di screening anti-HCV in Italia. PharmacoEcon, Ital Res Artic 2011;13(2):1-191590-9158/11/0002-0001/\$49.95/0.

30. Wolfe RA, Roys EC, Merion RM. Trends in organ donation and transplantation in the United States, 1999-2008. Am J Transplant. 2010;10(4 Pt 2):961-72.

31. Camma C, Petta S, Enea M, Bruno R, Bronte F, Capursi V, Cicchetti A, Colombo GL, Di Marco V, Gasbarrini A, et al. Costeffectiveness of boceprevir or telaprevir for untreated patients with genotype 1 chronic hepatitis C. Hepatology. 2012;56(3):850-60.

32. Ravasio R. Costo efficacia di peginterferone $\alpha-2 \mathrm{a}+$ ribavirina versus peginterferone $\alpha-2 b+$ ribavirina nel trattamento dell'epatite cronica di tipo $\mathrm{C}$ in pazienti HIV co-infetti. PharmacoEcon, Ital Res Artic. 2008;10(1):37-47.

33. DRG 480: Age.n.as. Ricoveri ospedalieri-i sistemi tariffari regionali vigenti nell'anno 2009. Gennaio 2010.

34. Organisation for Economic Co-operation and Development (OECD). Annual Average Wage. http://stats.oecd.org/Index. aspx ? DataSetCode $=$ AV_AN_WAGE (ultimo accesso marzo 2014).

35. Calcolatrice di giorni lavorativi in Italia. www.giorni-lavorativi. com (ultimo accesso dicembre 2013).

36. Banca d'Italia-Il sito ufficiale della Banca Centrale Italiana. www.bancaditalia.it/ (ultimo accesso dicembre 2013).

37. INPS. L. 104/1992 art. 33-Dlgs 151/2001 art. 42. Permessi retribuiti legge 104. http://www.inps.it/portale/default.aspx? itemdir $=5941$ (ultimo accesso dicembre 2013).

38. Puoti C, Guarisco R, Spilabotti L, Bellis L, Mitidieri Costanza O, Dell' Unto O, Elmo MG. Should we treat HCV carriers with normal ALT levels? The ' $5 \mathrm{Ws}$ ' dilemma. J Viral Hepatitis. 2012;19(4):229-35.

39. Dore GJ, Ward J, Thursz M. Hepatitis C disease burden and strategies to manage the burden (Guest editors Mark Thursz, Gregory Dore and John Ward). J Viral Hepatitis. 2014;21(Suppl 1):1-4.

40. Bruggmann P, Berg T, Øvrehus ALH, Moreno C, Brandão Mello $\mathrm{CE}$, et al. Historical epidemiology of hepatitis $\mathrm{C}$ virus $(\mathrm{HCV})$ in selected countries. J Viral Hepatitis. 2014;21(Suppl 1):5-33. 